\title{
Prediction and Theoretical Investigation of the Morphology of Erythromycin Dihydrate Crystals
}

\author{
Yan Zhang \\ Key Laboratory of Food Nutrition and Safety (Tianjin University of Science and Technology), Ministry of Education, Tianjin \\ 300457, PR China \\ *For correspondence: Email: cpzyyan@126.com
}

\begin{abstract}
Purpose: To predict and theoretically investigate the morphology of erythromycin dihydrate crystals. Methods: The crystal morphology of erythromycin dihydrate was predicted using the Bravais-FriedelDonnay-Harker (BFDH) and attachment energy (AE) models of molecular simulation software, Cerius ${ }^{2}$, in vacuo.

Results: The morphology predicted by the two models is approximately consistent. The morphology predicted by $A E$ model was in good agreement with the morphology of crystals grown from solution. The main crystal faces $\{002\},\{101\},\{011\}$ and $\{012\}$ were observed in the morphology predicted by $A E$ model. By cleaving revealable crystal faces in the morphology predicted by $A E$ model, surface chemistry visualization and theoretical analysis based on interaction of $\mathrm{H}$-band net formed in intramolecules or inter-molecules for important morphological forms were performed.

Conclusion: The results show that $\mathrm{H}$-band interaction plays a critical role in the plate-like morphology of erythromycin dihydrate, which is in accordance with the theory of Periodic Bond Chain (PBC).
\end{abstract}

Keywords: Erythromycin dihydrate, Morphology prediction, Theoretical investigation, Molecular simulation

Tropical Journal of Pharmaceutical Research is indexed by Science Citation Index (SciSearch), Scopus, International Pharmaceutical Abstract, Chemical Abstracts, Embase, Index Copernicus, EBSCO, African Index Medicus, JournalSeek, Journal Citation Reports/Science Edition, Directory of Open Access Journals (DOAJ), African Journal Online, Bioline International, Open-J-Gate and Pharmacy Abstracts

\section{INTRODUCTION}

The shape of a crystal is governed by the relative growth rates of each of the crystal faces present. The most prominent face of a crystal is the slowest growing, while the smallest face is the fastest growing [1]. The early work by Gibbs proposed that the shape of a crystal will be one to minimize the total free energy associated with the surface energies of the grown crystal. Later, Bravais et al [1,2] proposed that crystal is formed by faces bounding the directions of the greatest interplanar spacing of the crystal lattice based merely on crystal lattice geometry. In 1955, Hartman and Perdok proposed periodic-bond chain $(\mathrm{PBC})$ theory based on a strong bond between molecules running parallel to a crystallographic direction [1,3]. In 1980, based on PBC model, they proposed attachment energy model. Crystal growth was viewed as the attachment of slices or layers of ordered molecules to an existing crystal face [1], requiring the determination of the lattice and slice energies for calculation of the attachment energy in important crystallographic direction $[4,5]$.

Erythromycin is a mixture of macrolide antibiotics. Erythromycin A (Figure 1) is the main and active component and commercial erythromycin is usually available as the dihydrate [6]. Crystallization is effected via molecular recognition at the interface between the growing 
crystal and its mother phase. Such effects have been related to growth solution thermodynamics, specific face structure and growth mechanism, and molecular recognition process at specific planes [7].

Increased understanding of crystal growth can enhance the performance of the separation and purification processes in industries [8,9]. The aim of the present research is to apply advanced molecular modeling to erythromycin dihydrate to simulate the theoretical morphology. In the course of refining the model, valuable information concerning molecular interactions within the pure erythromycin dihydrate crystal was extracted from the simulation.

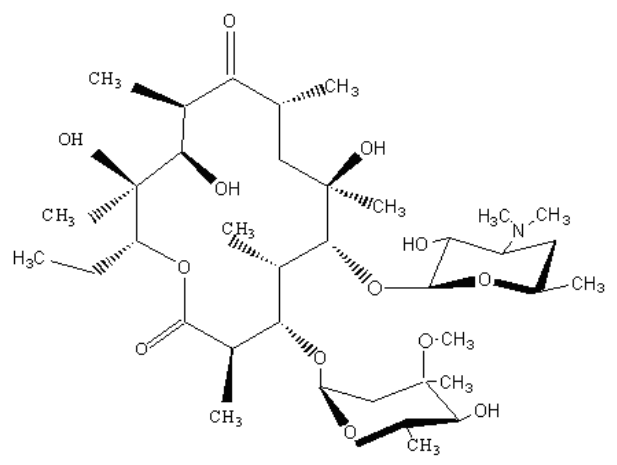

Figure 1: Molecular structure of erythromycin A

\section{EXPERIMENTAL}

\section{Materials and chemicals}

Erythromycin A dihydrate (Xi'an Rejoy Co Ltd, China), > $90.0 \%$ purity, molecular weight 769.94, molecular formula $\mathrm{C}_{37} \mathrm{H}_{67} \mathrm{NO}_{13} \cdot 2 \mathrm{H}_{2} \mathrm{O}$ ) was used for investigation. Recrystallization was performed to obtain erythromycin dihydrate. Distilled deionized water of HPLC grade was used.

\section{Scanning electronic microscopy (SEM) and microscopy measurement}

A microscopic observation of the crystal morphology was carried out taking advantage of scanning electronic microscopy (JSM-6700F Japan). Digital image analysis was performed using a Panasonic Lumix DMC-FZ20 system with the Panasonic image analysis connected to a 3CCD color vision camera mounted on an Olympus $\mathrm{BH} 2$ optical microscope.

\section{Cerius 2 modeling}

Crystal structures of erythromycin dihydrate were established by calculation of the simulated PXRD pattern. The program, TREOR90 of software Cerius $^{2}$, was used to collect frames of diffraction data, index reflections, and establish crystal cell parameters. For atomic charge assignments and energy minimizations, the Charge Equilibrium and Compass force field were used, respectively. Single crystal structure data of dihydrate was achieved by Cambridge Crystallographic Database and used to compare with the result of calculation of the simulated PXRD pattern.

\section{Crystal morphology prediction using BFDH and $A E$ model}

Classic molecular mechanics and dynamics simulations were carried out using the Cerius ${ }^{2}$ software package. The crystal builder, molecular mechanics, and morphology prediction modules of Cerius ${ }^{2}$ were employed to accomplish the modeling goals of this research. Gasteiger charge equilibrium and Dreiding force field were used. More detailed reviews of molecular mechanics and numerical minimization routines are as described previously $[10,11]$.

\section{RESULTS}

\section{Structure analysis}

The experimental PXRD pattern of erythromycin dihydrate was presented in Figure 2, which is consistent with previous reports. The PXRD pattern of dihydrate in Figure 2 also agrees well with the simulated pattern by single-crystal analysis from the Cambridge Crystallographic Database. The crystal structure of erythromycin dihydrate has been resolved using X-ray diffraction studies from which it was obtained that the unit cell shows a orthorhombic P212121 space group with $a=9.183 \mathrm{~A}^{\circ}, b=9.631 \mathrm{~A}^{\circ}, c=$ $47.151 \mathrm{~A}^{\circ}$.

In our simulations, we obtained the unit cell structure of the erythromycin dihydrate crystal from the Cambridge Crystallography Data Bank. The structure of unit cell of erythromycin dihydrate crystal was shown in Figure 3.

\section{Morphology prediction by BFDH and $\mathrm{AE}$ model}

The three faces $\{002\},\{011\},\{101\}$ dominated the crystal habit as revealed from the BFDH morphological prediction.

The results are summarized in Table 1. According to BFDH law, the larger the interplanar distance $d_{h k l}$ is, the larger the morphological importance (MI) of the corresponding $h k l$ face is. From the Table 1 , it can be seen that $d_{h k l}$ of $\{002\}$ 
face is the largest and its facet area is $53.27 \%$ of total facet area, which means $\{002\} \square$ face owns the largest the morphological importance (MI). The $d_{h k l}$ of $\{011\} \square$ face is slightly larger than $\{101\}$ face and $d_{h k l}$ of $\{110\} \square$ is the smallest.

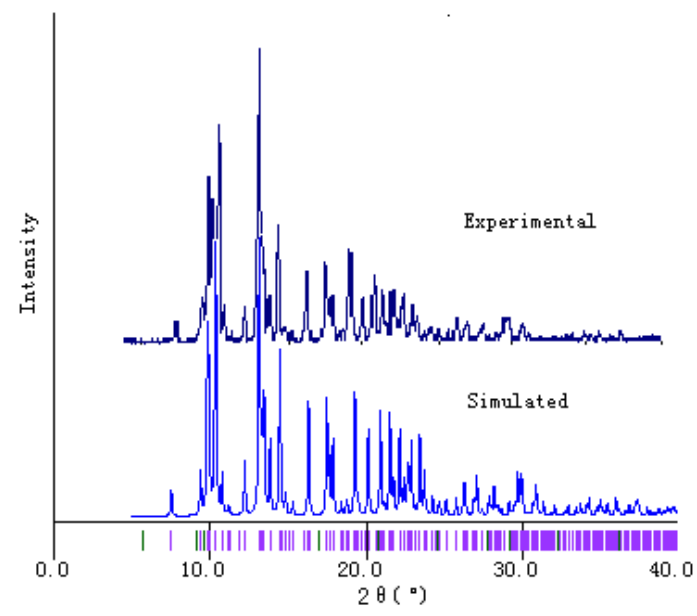

Figure 2: Overlay of experimental (top) and simulated (bottom) XRPD patterns of erythromycin dehydrate

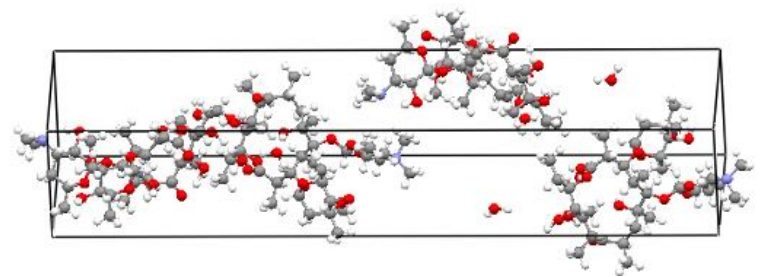

Figure 3: Crystal structure of orthorhombic erythromycin dihydrate

The results of attachment energy calculations are reported in Table 2. Based on attachment energy model, Ml sequence is based on the assumption that the growth rates of faces are proportional to attachment energy. Hence, the greater attachment energy, the faster the corresponding face growth and the smaller its morphological importance. Once the relative growth rates of the significant faces are known, a macroscopic image of the crystal morphology can be postulated.

\section{DISCUSSION}

Crystal morphology predicted by BFDH model can be deduced and was shown in Figure 4(a), revealing all faces which belong to the crystallographic zones $\{002\},\{011\},\{101\}$ and $\{110\}$, respectively. From Figure $4(a)$, there is a well agreement with calculation result in Table 1. The $\{002\} \square$ face was found to be the largest in area, subsequently $\{011\}$ face, $\{101\}$ face and $\{110\}$ face.

Table 1: Calculated results based on BFDH model

\begin{tabular}{|c|c|c|c|c|}
\hline hkl & Multiplicity & $d_{h k l}$ & $\begin{array}{l}\text { Total } \\
\text { facet } \\
\text { area }\end{array}$ & $\begin{array}{c}\text { Total } \\
\text { facet } \\
\text { area }(\%)\end{array}$ \\
\hline$\left\{\begin{array}{lll}0 & 0 & 2\end{array}\right\}$ & 2 & 23.58 & 833.9 & 53.27 \\
\hline$\left\{\begin{array}{lll}0 & 1 & 1\end{array}\right\}$ & 4 & 9.437 & 370.0 & 23.64 \\
\hline$\left\{\begin{array}{lll}1 & 0 & 1\end{array}\right\}$ & 4 & 9.014 & 351.5 & 22.45 \\
\hline$\{110\}$ & 4 & 6.646 & 10.16 & 0.6487 \\
\hline
\end{tabular}

Crystal morphology predicted by AE model can be deduced and was shown in Figure 4(b). The morphology (Figure 4a) predicted by BFDH model is approximately consistent with that predicted AE model. The main differences exist in disappearance of $\{110\}$ face and appearance of $\{012\} \square$ face in $A E$ model. From Table 2 and Figure 4(b), it can be seen that there is a good correlation between growth face area and the attachment energy of the dominant face. The morphology predicted is plate-like, suggesting that a single face is indeed dominating the overall crystal growth. In these cases, the most dominant face has an attachment energy whose absolute value is much lower than that for the other faces, and the relative growth rate is notably smaller. Hence, one face dominates the crystal growth.

\section{Comparison of predicted morphology with experimental growth crystal}

Morphological simulations were confronted with experimental data obtained from solution grown crystals. Crystal morphology was assessed by scanning electronic microscopy and optical microscopy. Morphological sketch was shown in Figure 5(a) and 5(b). The predicted morphology based on $A E$ and BFDH models compares favorably with the experimental morphology. The main difference between the experimental morphology and predicted morphology is that

Table 2: Calculated results based on AE model

\begin{tabular}{|c|c|c|c|c|c|}
\hline hkl & Multiplicity & $d_{h k l} / \AA$ & $\mathrm{E}_{\text {att }}($ total $) /(\mathrm{kJ} / \mathrm{mol})$ & $\begin{array}{c}\text { Total facet } \\
\text { area/ } \AA\end{array}$ & $\begin{array}{c}\text { Total facet } \\
\text { area } \% / \AA\end{array}$ \\
\hline$\{002\}$ & 2 & 23.58 & 42.16 & 89580 & 53.55 \\
\hline$\{011\}$ & 4 & 9.014 & 102.6 & 42380 & 25.33 \\
\hline$\{101\}$ & 4 & 9.437 & 125.7 & 21215 & 12.68 \\
\hline$\{110\}$ & 4 & 8.557 & 123.6 & 14118 & 8.439 \\
\hline
\end{tabular}




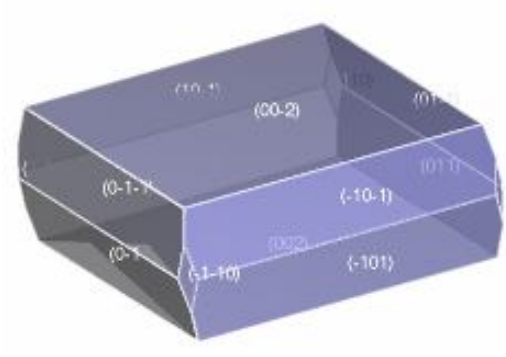

(a)

Figure 4: Predicted crystal morphology (a)

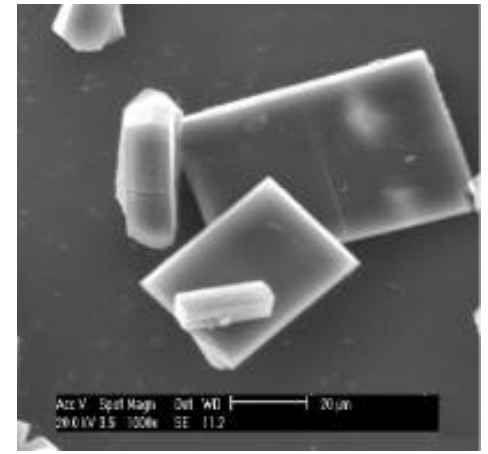

(a)

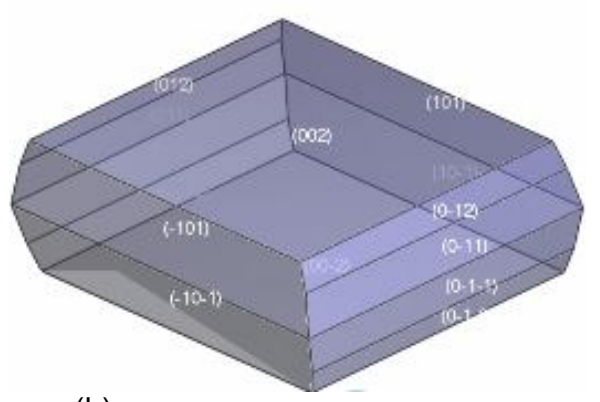

(b)

(a) BFDH model, (b) AE model

Figure 5: Experimental crystal morphology

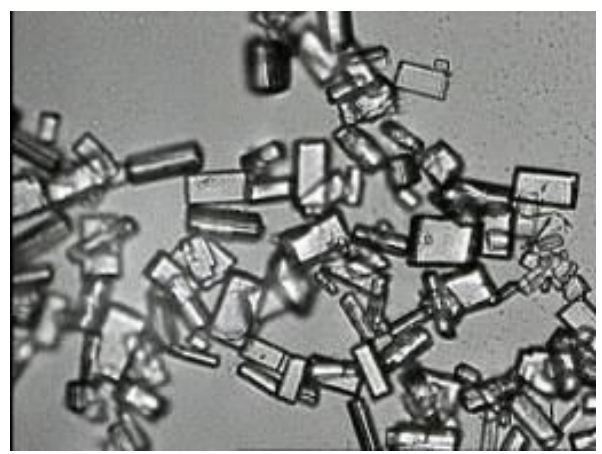

(b)
\{110\} face disappears in the experimental morphology, resulting in a squarer plate, which is more consistent with the morphology predicted by $A E$ model than by BFDH model. Despite difference between predicted and experimental morphology, it should be remembered that, overall, the predicted and solution grown morphology are rather comparable. By further analyzing morphology predicted by two models and experimental morphology, it can be seen that $\square 002 \square \square$ face is the most dominant in area. This was perhaps due to the fact that this face had no growth promoting hydrogen-bonding component involved in the intermolecular interactions involved in its attachment energy, in contrast to those involved on the $\{101\},\{011\}$ and $\{012\}$ faces.

\section{Surface chemistry visualization for important morphological forms}

The surface chemistry of the four surfaces was investigated: the four faces $\{011\},\{101\},\{012\}$ and $\{002\}$ expected dominate the crystal habit as revealed from the AE morphological prediction.

The results are summarized in Figure 6 . The $\{011\}$ surface (Figure 6a) was found to be very open and rough on the molecular level revealing a diagonal pattern down through the surface with alternating orientations of the erythromycin molecule, to allow for the possibility of hydrogen bonding with appropriate molecules. In addition, with this surface, both water molecule and hydroxyl components of the erythromycin molecule were found to be revealable to possible binding with appropriate molecules.

Examination of the $\{101\}$ surface (Figure 6b) revealed a rough surface topography on the molecular level with hydrogen bonding being found to be present diagonally down the surface. Two orientations of the erythromycin molecule were observed, horizontally and vertically aligned to the surface, respectively.

The $\{012\}$ surface (Figure $6 c$ ) was found to be very smooth on the molecular level, allowing the exposure of only the hydrocarbon components of the erythromycin molecule. The pattern produced was diagonal in nature with hydrogen bonding and donor or acceptor atoms were active on the surface for binding on coming molecules.

The $\{002\}$ surface (Figure $6 d$ ) was found to be rough on the molecular level. In contrast to the previous three cases, hydrogen bonding was found to run parallel with the $\{002\}$ face. Only the amino components were found to be exposed on the surface. 


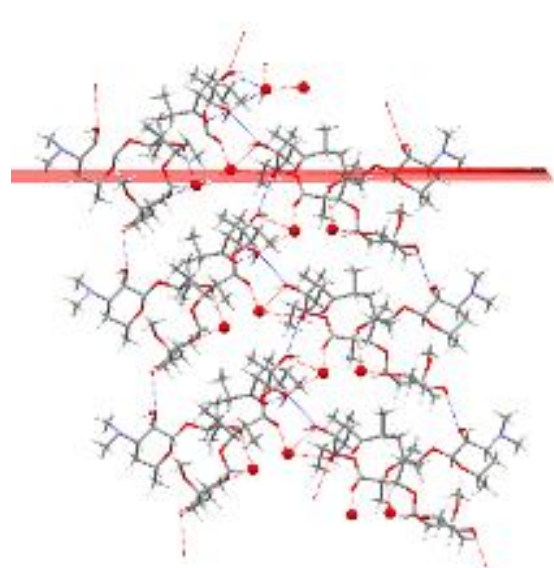

(a)

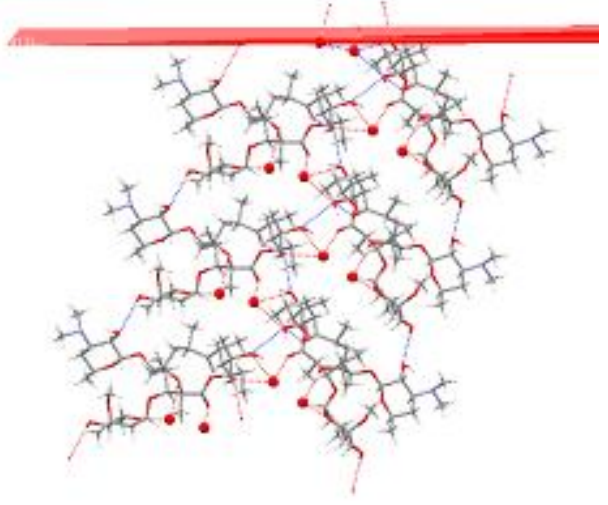

(c)

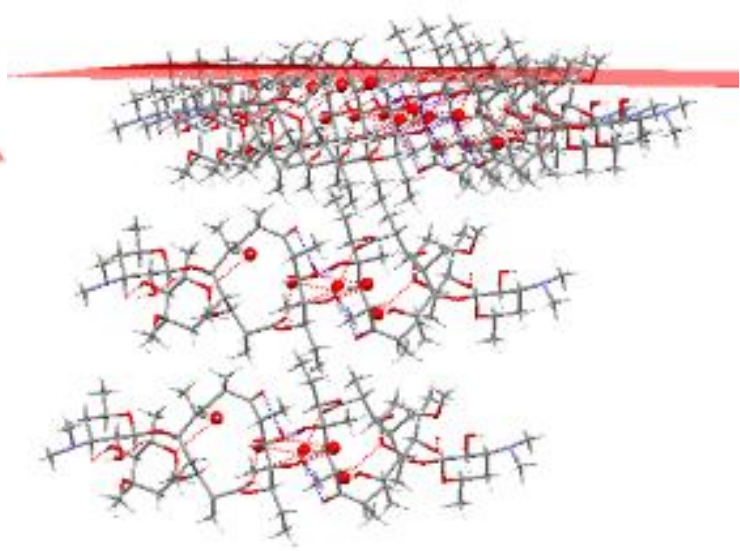

(b)

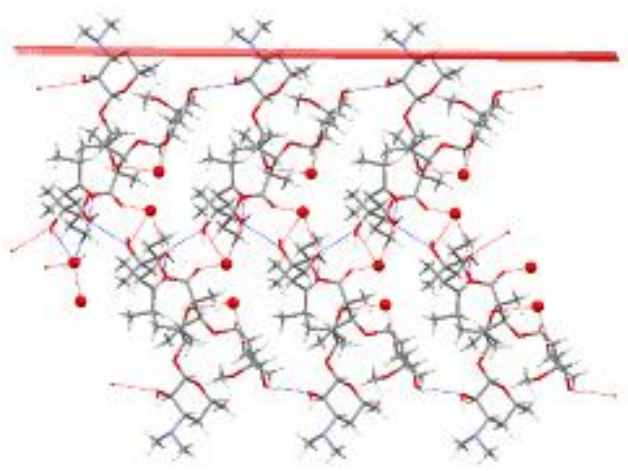

(d)

Figure 6: Cleavage structure of main exposure crystal plane (a) $\{011\}$, (b) $\{101\}$, (c) $\{012\},(d)\{002\}$

\section{PBC theory analysis}

According to PBC theory, a PBC is a strong bond between molecules running parallel to a crystallographic direction. Growth rate is related to direction of bond chain. The fastest direction of crystal growth is that of the strongest chemical bond present.

It is for $\{011\},\{101\}$ and $\{012\}$ faces that there are the hydrogen bonds directing these surfaces to position of erythromycin growth units on these surfaces, thus resulting in an enhanced growth rate of these faces. However, on the $\{200\}$ face where growth does not involve the formation of crossing hydrogen bonds, growth rate of this face is small. The fact that the $A E$ modeling has faithfully predicted this result may be a reflection on the capabilities simulating the difference in attachment energies between faces growing with and without hydrogen bonding.

\section{CONCLUSION}

The crystal morphology of erythromycin dihydrate has successfully been predicted using
AE model in conjunction with BFDH model. Accurate modeling and morphology prediction of this crystal are achieved. The morphology predicted by two models is approximately consistent. The morphology predicted by $A E$ model is more consistent with the morphology of crystals grown from solution than BFDH model. The main crystal faces $\{002\},\{101\},\{011\}$ and $\{012\}$ are observed and dominant in morphology predicted by $A E$ model. By cleaving these dominant crystal faces, surface chemistry visualization and theoretical analysis based on interaction of $\mathrm{H}$-band net formed in intramolecules or inter-molecules for the important morphological forms are performed. The result indicates that $\mathrm{H}$-band interaction plays a critical role for the plate-like morphology of erythromycin dihydrate, which is in accordance with the theory of Periodic Bond Chain (PBC).

\section{REFERENCES}

1. Givand JC, Rousseau RW, Ludovice PJ. Characterization of L-isoleucine crystal morphology from molecular modeling .J Cryst Growth, 1998; 194: 228-238. 
2. Prywer J. Explanation of some peculiarities of crystal morphology deduced from the BFDH law .J Cryst Growth, 2004; 270: 699-710.

3. Pfefer G, Boistelle R. Theoretical morphology of adipic acid crystals .J Cryst. Growth, 2000; 208: 615-622.

4. Hartman $P$, Bennema $P$. The attachment energy as a habit controlling factor: I. Theoretical considerations .J Cryst Growth, 1980; 49: 145-156.

5. David S, Coombes C, Richard A. Calculation of Attachment Energies and Relative Volume Growth Rates as an Aid to Polymorph Prediction .Cryst Growth Des, 2005; 5: 879-885.

6. Wang ZZ, Wang JK, Dang LP, Zhang MJ. Crystal Structures and the Solvent-Mediated Transformation of Erythromycin Acetone Solvate to Dihydrate during Batch Crystallization .Ind Eng Chem Res, 2007; 46(6): 1851-1858.
7. Elaine MW, Kevin JR, Stephen JM. A Molecular Dynamics Study of Solvent and Impurity Interaction on the Crystal Habit Surfaces of $\varepsilon$-Caprolactam .Langmuir, 1998; 14: 5620-5630.

8. Kubota N, Mullin JW. A kinetic model for crystal growth from aqueous solution in the presence of impurity.J Cryst Growth, 1995; 152: 203-208.

9. Koolman HC, Rousseau RW. Effects of isomorphic compounds on the purity and morphology of Lisoleucine crystals .AIChE Journal, 1996; 42:147-153.

10. Linert W, Renz F. Modeling crystal structures by numerical methods: a tool for practical structure analysis .J Chem Inf Comput Sci, 1993; 33(5): 776781.

11. Allen MP, Tildesley DJ. Computer Simulation of Liquids, Oxford Science Publications, New York, 1996. 